\title{
Improving the Compliance to the Use of Venous Thromboembolism (VTE) Prophylaxis in Medical Services Department Hospitals, Riyadh, Saudi Arabia
}

\section{Yasser Alotaibi ${ }^{1}$, Maha Bassim ${ }^{2}$, Noura Alnowaiser ${ }^{3}$, Mohamed Nassif ${ }^{4}$, Amal Al-Gosi ${ }^{5}$ and Mostafa Kofi ${ }^{5 *}$}

${ }^{1}$ Family Physician, Continuous Quality Improvement and Patient Safety, Medical Services General Directorate, Riyadh, Kingdom of Saudi Arabia

${ }^{2}$ Consultant and Senior Trainer in Family Medicine, Medical Doctorate degree in Family Medicine, Department of Family and Community Medicine at PSMMC, Riyadh, Kingdom of Saudi Arabia

${ }^{3}$ Director of Continuous Quality Improvement and Patient Safety Department, Director SMO At Health Services General Directorate, Ministry of Defense, Senior Consultant Family Medicine, Member of Executive Committee for Saudi Center of Accreditation for Healthcare Institutions (CBAHI), Member of Board of Directors for Saudi Patient Safety Center, International JCI Consultant, Kingdom of Saudi Arabia

${ }^{4}$ Physician with Master Degree in Healthcare Quality, Public Health, Ain Shams University, Egypt and Master Degree in Business Administration, Edinburgh School of Business, Heriot Watt University, Scotland and NAHQ Certified Professional in Healthcare Quality CPHQ, NPSF/IHI Certified Professional in-patient Safety CPPS and Lean Six Sigma Green Belt, Currently Working as Healthcare Quality Expert in Health Services Directorate of Ministry of Defense in Saudi Arabia, Kingdom of Saudi Arabia

${ }^{5}$ Consultant, Family and Community Medicine Department, Prince Sultan Military

Medical City, Riyadh, Kingdom of Saudi Arabia

*Corresponding Author: Mostafa Kofi Consultant, Family and Community Medicine Department, Prince Sultan Military Medical City, Riyadh, Kingdom of Saudi Arabia.
Received: October 18, 2021

Published: November 17, 2021

(C) All rights are reserved by Mostafa Kofi., et al.

\begin{abstract}
Introduction: VTE is a common preventable complication for hospital bed stay and responsible for a huge loss in costs and deaths. Despite the MDS required target of 95\% for compliance to the implementation of VTE thrombo-prophylaxis; unfortunately; the current status is not the case.

Methods: The Institute for Health Care Improvement (IHI) model of health care improvement was used. Pre-post implementation of VTE prophylaxis program was implemented in 2020 compared to 2017 at Medical Services Department (MSD) hospitals comparison of KPI before and after was analyzed using descriptive and analytic statistics.

Results: The average compliance for VTE prophylaxis in 21 MSD hospitals was $80 \%$ in the second quarter 2017 with 5 hospitals performing below $80 \%$ two of them performed below 40\%. Also, Increase the compliance to the VTE prophylaxis in MSD Hospitals, to reach the target $95 \%$ within 12 months. Study of different interventions strategies effectiveness used by the hospitals for improving the use of VTE prophylaxis; led to improve compliance with CIBHI standard.
\end{abstract}


Improving the Compliance to the Use of Venous Thromboembolism (VTE) Prophylaxis in Medical Services Department Hospitals, Riyadh, Saudi Arabia

Conclusion: The quality improvement project for 5 MSD hospitals, showed a statistically significant difference for improvement after implementing VTE prophylaxis improvement program from $70 \%$ to $99 \%$ during the project study duration.

For the desired outcomes, the intervention strategies have to be strong and multilayered such as, implementing VTE prophylaxis policy, Leadership involvement, linking VTE prophylaxis compliance to the annual physician evaluation, frequent auditing, personal feedback, training and education

Keywords: VTE; Health Care Research; Quality Improvement

\section{Introduction}

\section{Problem description}

Implementing VTE prophylaxis is one of the ESR CBAHI Amaan program standards required to be fulfilled by all hospitals in Saudi Arabia. And one of MSD KPI. The compliance for VTE in MSD hospitals should be $95 \%$ or more.

Venous thromboembolism (VTE) is a frequent dangerous complication occurring during hospital treatment with total annual incidence of 70-200 per 100,000 citizens. Venous thromboembolism includes deep vein thrombosis (DVT) and pulmonary embolism (PE). Pulmonary embolism is responsible for about $10 \%$ of hospitalization-related deaths and is the most common avoidable reason for deaths in hospitals [12].

The average compliance for VTE prophylaxis in 21 MSD hospitals was $80 \%$ in the second quarter 2017 with 5 hospital performing below $80 \%$ two of them performed below $40 \%$.

\section{Available knowledge}

Hospitalized acute medically ill patients are vulnerable to venous thromboembolism (VTE), known as hospital-acquired thrombosis (HAT). The elevated risk of HAT is usually due to a combination of factors, with immobility and a prothrombotic state due to acute illness being the most frequent. These HAT events may be fatal, and patients who survive the initial event may be subject to VTE recurrence, chronic discomfort from post-thrombotic syndrome and, although rare, may develop chronic thrombo-embolic pulmonary hypertension, which is often debilitating [11]. The risk of HAT can be reduced with effective thromboprophylaxis. Venous thromboembolism (VTE) includes DVT and PE. DVT is a major preventable cause of mortality and morbidity worldwide. DVT and PE account for 60,000 to 100,000 deaths annually in the United States [1].

Hospitalized patients are at increased risk of developing DVT (approximately 50\%), and this increases the risk of PE [1].

Pulmonary embolism is one of the most common, but preventable, causes of death in hospitalized patients [2].
Only $50 \%$ of the hospitalized patients receive DVT prophylaxis [2].

DVT increase the risk of pulmonary embolism by $50 \%$ and also leads to the post-thrombotic syndrome [1].

Using DVT prophylaxis in hospitalized patients decrease the risk of DVT anywhere from $10 \%$ to $80 \%$. DVT prophylaxis decreases the risk of DVT/PE in both hospitalized medical and surgical patients. However, mortality benefits have been reported in surgical patients but not in medical patients [3].

Three Examples of Effective Implementation of VTE prophylaxis and Clinical Decision Support: The Johns Hopkins collaborative team, The University of Michigan, and Banner Good Samaritan Regional Medical Center they used the following consequently.

- Reinforcing appropriate prophylaxis through staff engagement, education, regular evaluation, good clinical decision support in order sets, and layered interventions to reinforce the protocol.

- $\quad$ Targeting all adult inpatients, adding forcing functions with hard stops to guarantee a risk assessment was done, using algorithmic logic, grouping risk factors.

- The comprehensive deployment in reinforcing the medical center's VTE prevention protocol.

These interventions were

Leadership involvement, linking VTE prophylaxis compliance to the annual physician evaluation, frequent auditing, personal feedback, and included the VTE prophylaxis in the pre- admission papers.

Rationale

- VTE is one significant cause of preventable hospital related mortality/morbidity

- Indications that incidence is under-estimated

- $\quad$ May be under-reported [2]

- 2013 Quality Systems Assessment - around 40\% of pa- 
tients are assessed for VTE risk at admission, and only $70 \%$ of those at risk are provided with appropriate prophylaxis

- $\quad$ Consistent with international studies which found that only 30 to $50 \%$ of patients at risk receive appropriate prophylaxis [3].

- $\quad$ Medical Service Department (MSD) at the ministry of defense general directorate Saudi Arabia is supervising the ministry of defense hospitals.

- Despite the MDS required target of 95\%, for compliance to the implementation of VTE thorombo prophylaxis, The average compliance for VTE prophylaxis in 21 MSD hospitals was $80 \%$ in the second quarter 2017 with 5 hospital performing below $80 \%$ two of them performed below $40 \%$.

- The cost of a single episode of VTE is estimated to be more than 15000 US Dollar [3].

\section{Objectives}

- Increase the compliance to the VTE prophylaxis in MSD Hospitals, to reach the target $95 \%$ within 12 months.

- Study different interventions strategies effectiveness used by the hospitals for improving the use of VTE prophylaxis.

- Improve compliance with CIBHI standard.

\section{Methods}

Context

- $\quad$ The quality department in MSD, had identified the hospitals which performed below the required standard.

Study design

The project time table from June 2018 to June 2019

- Two randomly selected, hospitals from the identified low performance regarding VTE prophylaxis hospitals were selected as a pilot.

- $\quad$ Piloting a new VTE risk assessment form was suggested by the governance (MSD), from the perspective that the current form was not user friendly.

- Incorporating order set, and prophylaxis assessment form to standardize the practice through evidence-based guidelines was adopted.
- Order sets helped in reducing variation and unintentional oversight through standardized formatting and clear presentation of orders that would result in preventing or minimize errors of omission, provide clinical decision support, and enhance providers' ordering efficiency.

- $\quad$ Based on the international journal of cardiovascular and cerebrovascular disease published $\left(1^{\text {st }}\right.$ September 2013) The identified barriers to compliance with clinical practice guidelines were taken into consideration during planning the interventions.

The quality department in charge or designee was selected as a project team leader for each hospital.

- $\quad$ Effective implementation of the VTE prevention protocol addresses the first four failure modes as discussed;

- No standardized protocols or order sets for VTE prevention exist.

- $\quad$ Order sets and prompts that reference VTE prevention are in place, but they provide inadequate guidance.

- $\quad$ Order sets with guidance are in place, but the order set is bypassed or not used.

- $\quad$ Order sets with guidance are in place and used, but used incorrectly [7].

The scale up of the project was based on the IHI collaborative model

- A focus group from the selected hospitals were identified.

- A project follow up timeline was developed as the following (3 learning session in total). One learning session every 3 month, where the selected participants will come to MDS head quarter to present their PDCA outcome.

Plan for the next PDCA and share other hospitals planning and implementation experience

- Video conference with all the involved hospitals to follow the implementation of the action plan every 6 weeks

- $\quad$ A driver diagram was developed (Attached)

- $\quad$ First learning session was conducted

- $\quad$ First learning session: At MSD to oversee the following per hospital.

First action plan (PDCA cycle) development by every hospital enrolled in the improvement program 
- The prophylaxis form for VTE was discussed to be piloted in the hospitals along with order set and policy.

Second learning session: At MSD to oversee the following per hospital

- Implement fist action plan (PDCA).

- Study the action plan outcomes

- Identify implementation barriers

- Identify lesson learned

- $\quad$ And develop second PDCA cycle.

Action taken

- $\quad$ Finalize the policies and order set

- $\quad$ Adopt risk assessment form for VTE, and piloting it in medical wards.

- $\quad$ Perform regular meeting for VTE Committee and create a term of reference to be reported to the ESR.

Committee

- Full implementation of the VTE risk assessment form, hospitals wide in medical and surgical wards.

- $\quad$ Physicians and nurses education sessions.

Third learning session: At MSD to oversee the following per hospital;

- Implement second action plan (PDCA).

- Study the action plan outcomes

- Identify implementation barriers

- Identify lesson learned

- $\quad$ And develop third PDCA cycle.

Action taken

- Monitoring of the implementation of VTE prophylaxis in the hospital

- Study the implementation barriers and implement the third PDCA for improvements

Target population

All 18 and over years old hospital admissions for medical and surgical departments for the selected hospitals.
Inclusion and exclusion criteria

Inclusion

All over 18 years old admitted to the hospital with the ICD principal diagnosis except (mental health, Obstetric, knee arthroplasty, hip arthroplasty, stroke) with patients stayed in the hospital for 2 or more days.

Exclusion

- $\quad$ Patients less than 18 years old

- $\quad$ Patients with length of stay less than 2 days

- $\quad$ Patients who are directly admitted to ICU

- $\quad$ Patients with ICD principal diagnosis code of mental illness, obstetric, stroke or knee and hip arthroplasty.

\section{Working definitions}

Percentage of patients who receives venous thromboembolism (VTE) prophylaxis on the day of or the day after hospital admission or surgery end date or for surgeries that start the day of or the day after hospital admission over the study period.

Data collection form

Excel sheet.

Data management plan

All data will be collected, retrospectively from the patient records, and daily fed to the excel for the selected hospital, data analysis per hospital based on the project variables, age, VTE prophylaxis conduction, VTE prophylaxis, type of improvement intervention.

Sampling methods

Cluster random sampling (cluster per unit).

Sample size calculation

- 6-77 include all 78-390 include 78

- 390 include $20 \%$ of total population.

Interventions

The change package consisted of the following

- Adoption of an evidence based VTE prophylaxis guidelines

- $\quad$ Standard VTE prophylaxis order set

- $\quad$ Staff training

Citation: Mostafa Kofi., et al. "Improving the Compliance to the Use of Venous Thromboembolism (VTE) Prophylaxis in Medical Services Department Hospitals, Riyadh, Saudi Arabia". Acta Scientific Medical Sciences 5.12 (2021): 104-111. 
Improving the Compliance to the Use of Venous Thromboembolism (VTE) Prophylaxis in Medical Services Department Hospitals, Riyadh, Saudi Arabia

- Daily follow up, and monthly control chart presentation for the compliance for VTE prophylaxis implementation for the hospital.

- Reinforce multidisciplinary team action (physicians, nurses, Quality improvement representative, IT, Related Departments heads, and Hospital administration).

- Include VTE prophylaxis implementation to the annual physician appraisal

- $\quad$ Review action plan with different intervention strategy against the VTE prophylaxis compliance percentage.

Process measure

- VTE prophylaxis compliance.

- $\quad$ Study the special cause for VTE prophylaxis improvement against the intervention in action plan used.

\section{Measures}

- VTE prophylaxis checklist was adopted, the implementation and completeness of the forms was audited on a monthly bases (any incomplete form will be considered as no compliance with VTE prophylaxis) bases for all inpatient adult patients in medical and surgical hospital departments.

- Quarterly PDCA cycles was generated and implements [3].

Cycles

- The measure was recorded in a control chart to assess the compliance to the improvement process.

- $\quad$ The special cause in compliance was studied.

Analysis

- Qualitative and quantitative methods used to draw inferences from the data

- Data was recorded on a control chart to detect special cause variation if any.

- $\quad$ Special causes were studied and causes had been recorded.

Ethical considerations

- Patient information would be anonymous,

- Patients will not be exposed to any harm.
- Approval of MSD general director for conducting the improvement project was obtained.

- Institutional review Board: This research is a quality improvement project, with no intervention to patients, has no risk to patients, no risk for patient confidentiality. So, this research need no IRB approval.

Data validation

Data was validated using different reviewers and by using different sources of data. (admission office papers, review patient records).

Results

Average Percentage Compliance Before \& After PI Project Across 6 MSD Hospitals

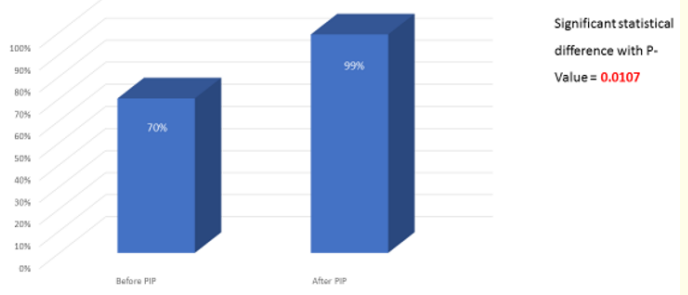

Figure 1: Before and after the quality improvement project for 5 MSD hospitals, which shows a statistically significant difference for improvement in implementing VTE prophylaxis from $70 \%$ to 99\% during the project study duration.

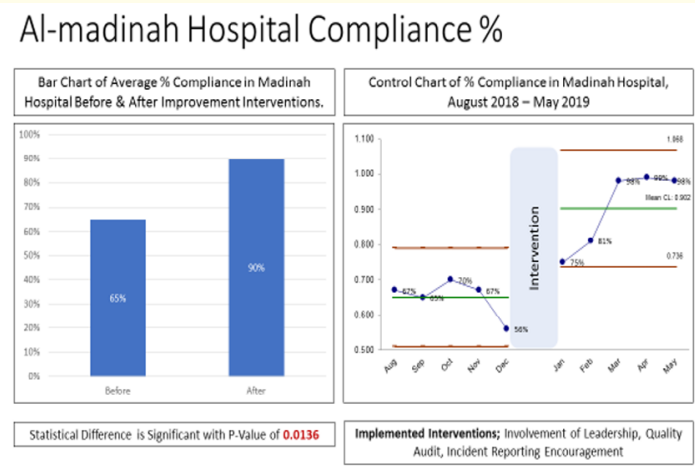

Figure 2: Almadina hospital which shows significant statistical improvement from $65 \%$ to $90 \%$. The special cause was the interventions they implement starting August 2018 which were (leadership involvement, frequent audit, incident reporting). 


\section{Sharourah Hospital Compliance \%}

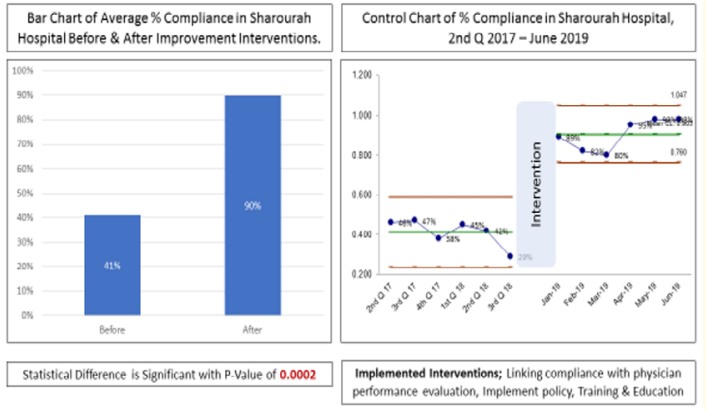

Figure 3: Sharourah hospital which shows significant statistical improvement from $41 \%$ to $90 \%$. The special cause for the improvement were the interventions they implemented started June 2018 (Linking compliance with physician performance evaluation, Implement policy, Training and Education).

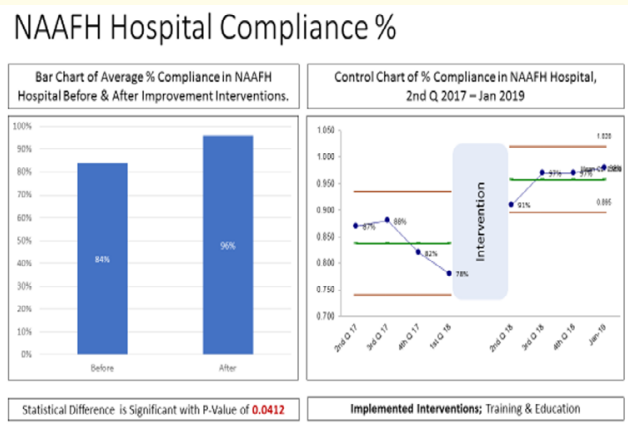

Figure 4: NAAFH hospital which shows significant statistical improvement from $84 \%$ to $96 \%$. The special cause for the improvement was the interventions they implemented June 2018which was (training and education).

NWAFH Hospital Compliance \%

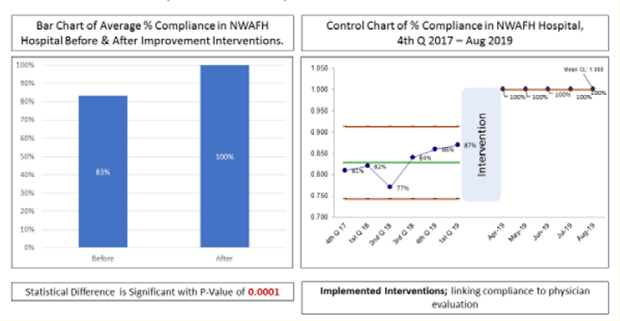

Figure 5: NWAFH hospital which shows significant statistical improvement from $83 \%$ to $100 \%$. The special cause for the improvement was the interventions they implemented January 2019 which was (linking compliance to physician evaluation).
KFMMC Hospital Compliance \%

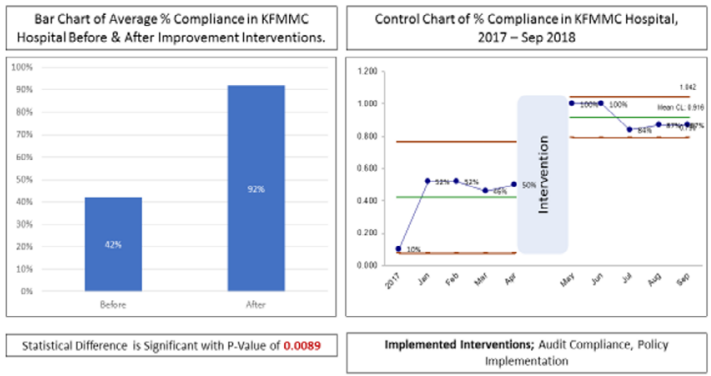

Figure 6: KFMMC hospital which shows significant statistical improvement from $42 \%$ to $92 \%$.

The special cause for the improvement was the interventions they implemented June 2018 which were (Audit Compliance, Policy Implementation).

Frequently used intervention is explained in table 1; This table showed the frequency of different used intervention strategies which were; leadership involvement, policy implementation, linking compliance to yearly physician evaluation, training, education, audit and incident reporting; so, the health care improvement (IHI) model for improvement was used is explained in figure.

\begin{tabular}{|l|c|}
\hline Leadership involvement & 1 \\
\hline Policy implementation & 2 \\
\hline $\begin{array}{l}\text { Linking compliance to the } \\
\text { yearly physician evaluation }\end{array}$ & 2 \\
\hline Training and education & 2 \\
\hline Audit & 2 \\
\hline Incident reporting & 1 \\
\hline
\end{tabular}

Table 1: Frequently used intervention.

\section{Discussion}

VTE is a major health problem as complication during patients' hospitals admission, with consequent Costs and burden to Health care system, also increased burden of illness to patients' possible disabilities. So, this Quality improvement Project was conducted to reduce risks to patients in terms of morbidity and mortality also to reduce burden on Health care system.

Adopting and Implementing the health care improvement (IHI) model for improvement facilitated the implementation and follow 
up of the quality improvement program for the selected hospitals. The importance of iterative (plan, do, study, act) PDSA cycles, helped to ensure that at each stage of the improvement cycle there was process optimization.

In order to improve patient safety, comply with the use of VTE prophylaxis and reaching the target, different improvement strategies were implemented and their effectiveness were measured. Based on the National Patient Safety Foundation, Three levels of intervention was mentioned, stronger interventions, intermediate interventions and weak interventions. Stronger interventions include those tasks which require less reliance on humans to remember to perform the task correctly) [6].

Several trials have been performed studying the use of single and multiple intervention strategies [5,8-10]. Single intervention such as passive dissemination of information was the least effective method.

This improvement project showed that multilayered PDCA follow up and combined strong system improvement interventions such as, policy implementation, audit, liking VTE prophylaxis implementation compliance to yearly physician evaluation improved the overall involved project hospitals compliance to the implementation of VTE prophylaxis. One hospital used single passive intervention strategy, which was education and although this hospital reached the target, this hospital basic performance level was higher than other hospitals.

But these results represented many challenges such as:

- Differences in hospitals health information system some hospitals use electronic system others still used paperwork system.

- Most of the hospitals used simultaneous multi-layered intervention strategies to improve compliance, which made it difficult to relate improvement to one specific intervention strategy.

- Staff turn over

- Outcome measured like VTE incidence, Pulmonary embolism, or cost reduction were not measured.

\section{Conclusion}

For quality improvement project desired outcomes, The suggested change in interventions strategies has to be strong, multilayered and Based on system improvement.
Policy implementation, Leadership involvement, linking improvement intervention compliance to the annual physician evaluation, frequent auditing, incident reporting system improvement are suggested

\section{Implications}

- The quality improvement project for 5 MSD hospitals, showed a statistically significant difference for improvement after implementing VTE prophylaxis from $70 \%$ to 99\% during the project study duration. MSD hospitals now are practicing VTE prophylaxis within the required target. Compliance with essential safety (ESR) standards that is required by CBAHI Amaan Program was achieved.

- One hospital used one intervention strategy, which was education and although the hospital reached the target but this hospital basic performance level was already high $84 \%$.

- For this improvement project, the implementation of multilayered strategies for intervention, such as policy implementation, linking VTE prophylaxis to physician yearly evaluation, Audit, incident reporting, were effective in improving compliance to the use of VTE prophylaxis.

\section{Bibliography}

1. Stone J., et al. "Deep vein thrombosis: pathogenesis, diagnosis, and medical management". Cardiovascular Diagnosis and Therapy 7 (2017): S276-S284.

2. Spyropoulos AC., et al. "The use of weighted and scored risk assessment models for venous thromboembolism". Thrombosis and Haemostasis 108.6 (2012): 1072-1076.

3. Collins R., et al. "Reduction in fatal pulmonary embolism and venous thrombosis by perioperative administration of subcutaneous heparin. Overview of results of randomized trials in general, orthopedic, and urologic surgery". The New England Journal of Medicine 318.18 (1988): 1162-1173.

4. The Breakthrough Series. "IHI's Collaborative Model for Achieving Breakthrough Improvement". Institute for healthcare improvement (2020).

5. "Revised Standards for Quality Improvement Reporting Excellence (SQUIRE 2.0)". September 15, 2015. Revised Standards for Quality Improvement Reporting Excellence (2015).

6. The National Patient Safety Foundation. Second online publication, Version 2, January 2016, RCA2 Improving Root Cause Analyses and Actions to Prevent Harm (2016). 
7. Internet Citation: Chapter 5. Implement the VTE Prevention Protocol. Content last reviewed February 2016. Agency for Healthcare Research and Quality, Rockville, MD (2013).

8. Alice G Bateman., et al. "The implementation of nice guidance on venous thromboembolism risk assessment and prophylaxis: a before-after observational study to assess the impact on patient safety across four hospitals in England". BMC Health Services Research 13.203 (2013).

9. Alexander T Cohen., et al. "Efficacy and safety of fondaparinux for the prevention of venous thromboembolism in older acute medical patients: randomised placebo controlled trial". BMJ 332.7537 (2006): 325-329.

10. Walter Ageno and Beverley J Hunt. "Reducing the burden of venous thromboembolism in the acute medically ill population with extended-duration thromboprophylaxis". The New England Journal of Medicine 365.23 (2011): 2167-2177.

11. Alina Wilkowska., et al. "Risk and prophylaxis of venous thromboembolism in hospitalized psychiatric patients. A review". Psychiatria Polska 52.3 (2018): 421-435.

Volume 5 Issue 12 December 2021

(C) All rights are reserved by Mostafa Kofi., et al. 